\title{
Modelo Demandas-Control-Apoyo social en el estudio del estrés laboral en el Perú
}

\section{Job demands-control-social support model in the study of labor stress in Peru}

\section{Sr. Editor:}

Los factores psicosociales en el trabajo (FPT) surgen de la interacción de las condiciones laborales, la experiencia y percepción del empleado y, tienen la posibilidad de afectar favorable o desfavorablemente la salud y el bienestar de los trabajadores, así como el desarrollo de las organizaciones. Una especial atención en los estudios de los FPT en los últimos años ha sido su configuración adversa o desfavorable denominada riesgos psicosociales en el trabajo (RPT) (1). En el contexto peruano, los RPT forman parte de la legislación laboral (Ley N²9783 de Seguridad y Salud en el Trabajo), que enfatiza la necesidad de realizar evaluaciones periódicas y la aplicación de estrategias preventivas para disminuir su efecto. Sin embargo, el estudio de los RPT todavía no es considerado en investigaciones peruanas, que se evidencia por la ausencia de artículos publicados sobre el particular, y por la falta de disciplinas como psicología de la salud ocupacional en los currículos universitarios (2), desconociéndose su impacto en el trabajador y en las organizaciones laborales. Por ello, se cree necesario incentivar estos estudios vislumbrando propuestas teóricas que guíen adecuadamente al investigador sobre el fenómeno de los RPT.

Desde esta perspectiva el modelo DemandasControl (MDC) (3), uno de los enfoques teóricos integrado al ámbito de la salud ocupacional, propone una explicación al proceso de estrés, motivación y aprendizaje en el trabajo, considerando las características psicosociales de las condiciones laborales y sus implicancias en la salud y en la productividad. La comprensión del MDC depende de términos clave como: a) Demandas psicológicas, relacionadas con las exigencias derivadas de la condición del trabajo (e. g., carga de trabajo, presión, ritmo, tiempo de trabajo, etc.), y b) control, que es un recurso personal integrado por la autonomía y

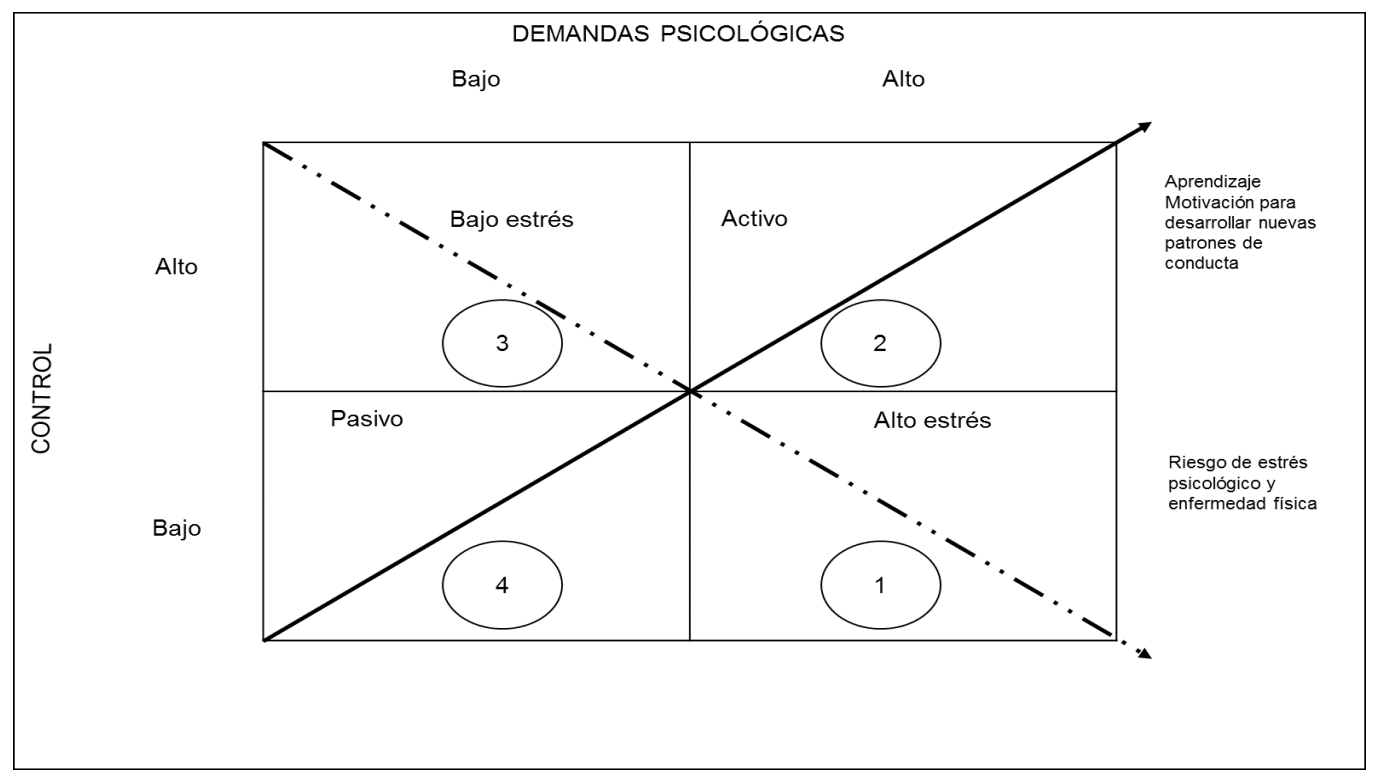

Figura 1. Modelo demandas psicológicas-control (4).

\footnotetext{
Instituto de Investigación, Escuela Profesional de Psicología, Universidad de San Martín de Porres. Lima, Perú.

a Psicólogo, Doctor en Educación;

b Bachiller en Psicología
} 
las habilidades del trabajador, que amortiguan las demandas psicológicas evitando la experiencia de estrés laboral.

En la figura 1, el MDC es ilustrado bajo cuadrantes integrados a las siguientes condiciones: a) Alto estrés: estrés laboral elevado con altas demandas y poco control que ocasiona fatiga, ansiedad, depresión y enfermedades físicas, b) Activo: estrés laboral moderado o bajo que comprende altas demandas y alto control que incide en la motivación y la búsqueda de soluciones en la actividad laboral, c) Bajo estrés: estrés laboral bajo, donde las bajas demandas y el alto control no generan tensión en el trabajador y, d) Pasivo: donde las bajas demandas y el bajo control ocasionan monotonía y falta de motivación en el empleado, impidiendo el desarrollo de las habilidades aprendidas.

Johnson y Hall (4), posteriormente proponen la tridimensionalidad del MDC integrando el concepto de apoyo social, recibiendo la denominación de Modelo Demandas Control Apoyo Social (MDCA). Los autores consideran que el apoyo social es una variable que disminuye la experiencia del estrés laboral y su ausencia está asociada con enfermedades coronarias, alto colesterol, altos niveles de enfermedad entre desempleados y un incremento del estrés laboral.

En la actualidad, el MDCA recibe soporte de sus supuestos teóricos debido a las evidencias de estudios empíricos (5), de igual forma se han elaborado instrumentos específicos para su estudio como el Cuestionario del Contenido del Trabajo (JCQ por sus siglas en inglés), el cual ha sido traducido en diversos países del mundo y cuenta con distintas versiones que constatan su utilidad.
La necesidad de realizar investigaciones asociadas al diagnóstico de los RPT y sus consecuencias, requiere de estudios empíricos que contemplen un marco teórico estructurado, coherente y de utilidad, como es el caso del MDCA, que sería una alternativa recomendable. De esta manera, el modelo podría guiar las investigaciones cuyos resultados permitirían tomar decisiones con respecto a la intervención o prevención pertinente en el entorno laboral explorado, incidiendo de esta forma, en la salud y el bienestar del trabajador.

\section{José Manuel Fernández-Arata, ${ }^{1, a}$, Gustavo Calderón-De la Cruz ${ }^{1, \mathrm{~b}}$}

\section{Correspondencia:}

José Manuel Fernández-Arata.

Av. Tomás Marsano 242, 5to piso. Lima, 34. Perú. Correo electrónico:mfernandeza1@usmp.pe Teléfono: 5115136300.

\section{REFERENCIAS BIBLIOGRÁFICAS}

1. Gil-Monte PR. Riesgos psicosociales en el trabajo y la salud ocupacional. Re Peru Med Exp Salud Pública. 2012; 29(2):237-241.

2. Fernández-Arata JM, Calderón-De la Cruz G, Navarro-Loli S. Psicología de la salud ocupacional: una especialidad emergente en el Perú. Rev Med Herd. 2016; 27:193-194.

3. Karasek R, Theorell, T. Healthy work: stress, productivity, and the reconstruction of working. New York: Basic Books; 1990.

4. Johnson JV, Hall EM. Job strain, workplace social support, and cardiovascular disease: a cross-sectional study of a random sample of the Swedish working population. American Journal of Public Health. 1988; 78:1336-1342.

5. Gonzáles NL, Juárez A, Camacho A, Noriega LH, Escobedo JP. Validez factorial y confiabilidad del cuestionario del contenido del trabajo (JCQ: Job Content Questionnarie) en empleados del sector salud, México. In: Juárez A, editors. Investigaciones psicométricas de escalas psicosociales en trabajadores mexicanos. Morelos: Plaza y Valdez Editores; 2016; p. 35-64. 